\title{
Orientações de enfermagem voltadas para o autocuidado de pessoas com insuficiência cardíaca
}

\author{
Nursing guidelines for the self-care of people \\ with heart failure
}

\section{Adryel Vieira Caetano da Silva' ${ }^{1}$ Beatriz Lucas de Carvalho ${ }^{2}$ (1) Maria Vilaní Cavalcante Guedes 3 (1)}

1'Autor para correspondência. Universidade Estadual do Ceará (Fortaleza). Ceará, Brasil. adryelvieira@hotmail.com 2,3Universidade Estadual do Ceará (Fortaleza). Ceará, Brasil. bbia.lluck@hotmail.com, vilani.guedes@uece.br

RESUMO | OBJETIVO: Identificar, na literatura científica, as principais orientações de enfermagem voltadas ao autocuidado de pessoas com insuficiência cardíaca. MATERIAIS E MÉTODO: Trata-se de uma revisão integrativa de literatura utilizando as bases de dados LILACS, MEDLINE, BDENF e a biblioteca virtual SciELO para a busca de publicações indexadas no período de 2009 a 2019, empregando os descritores 'insuficiência cardíaca', 'autocuidado' e 'enfermagem'. RESULTADOS: Com os 21 estudos da amostra, elencaram-se as recomendações primordiais voltadas para o autocuidado da insuficiência cardíaca: Adesão farmacológica; Monitorização de sinais, sintomas e peso corporal; Restrição hidrossalina; Atividade física; Hábitos alimentares; Conhecimento da insuficiência cardíaca; Vacinação; Suporte familiar; Consumo do álcool; Cessação do tabagismo; Repouso; Atividade sexual e Atividade laboral. CONCLUSÃO: Pontua-se a correlação direta da prática adequada do autocuidado com a redução significativa em taxas de reinternações e descompensação clínica da condição.

DESCRITORES: Enfermagem. Insuficiência cardíaca. Autocuidado. Cuidados de enfermagem.

\begin{abstract}
OBJECTIVE: To identify the main nursing guidelines for the self-care of people with heart failure in the scientific literature. MATERIALS AND METHOD: The present study is an integrative literature review based on LILACS, MEDLINE, BDENF databases and on the SciELO virtual library in order to search for resources published between 2009 and 2019, using the descriptors 'heart failure', 'self-care', and 'nursing'. RESULTS: Based on the 21 study samples, the presente study highlights the primary recommendations for the self-care of people with heart failure: Pharmacological adherence; Signals monitoring, symptoms and physical weight; Hydrosaline restriction; Physical activities; Eating habits; Knowledge of heart failure; Vaccination; Family support; Alcohol consumption; Smoking cessation; Rest; Sexual activity and Work activity. CONCLUSION: This study highlights the direct correlation between the appropriate self-care practice and the significative reduction in the rate of readmissions and the clinical decompensation of the condition.
\end{abstract}

DESCRIPTORS: Nursing. Heart failure. Self-care. Nursing care. 


\section{Introdução}

A insuficiência cardíaca (IC) é definida como a incapacidade funcional da bomba cardíaca no bombeamento de aporte sanguíneo necessário para atender às necessidades fisiológicas e metabólicas do organismo e, por ser caracterizada como via final da maior parte das doenças cardiovasculares, possui números elevados de hospitalizações e mortalidade ${ }^{1-2}$.

Segundo o Departamento de Informática do Sistema Único de Saúde (DATASUS), conforme os índices de morbimortalidade da insuficiência cardíaca, no período de 1996 a 2016, ocorreram 593.546 óbitos por IC³. Além do mais, é notório o crescimento anual das taxas de internações hospitalares evidenciado por mais de 2 milhões internações, no período de janeiro de 2008 a abril de 2018, conforme o Departamento ${ }^{4}$.

Com uma complexidade etiológica, numerosos fatores de risco tornam-se categóricos para o surgimento da IC tais como as condições: dislipidemia, Diabetes Mellitus (DM) e Hipertensão Arterial - e/ou hábitos de vida e comportamentais - tabagismo, etilismo, sedentarismo, obesidade e estresse que, ao se aliar a fatores genéticos, são os motivos para a ocorrência da condição ${ }^{5}$.

A sintomatologia característica da IC envolve dispneia/ortopneia, palpitações, síncope, dor torácica, dispneia paroxística noturna, fadiga, arritmias, edemas dos membros inferiores e outros ${ }^{6}$. Com o entendimento da detecção dos sinais e sintomas primordiais e dos fatores de risco, o cuidado de enfermagem surge como alicerce necessário à promoção da adesão terapêutica por meio do enfoque educativo e terapêutico evitando a readmissão hospitalar.

Os motivos apontados para os aumentos dos índices de hospitalizações envolvem o não seguimento correto da terapêutica prescrita farmacológica e não-farmacológicae/ouincapacidadedaidentificação de sinais e sintomas de descompensação da doença ${ }^{7}$. O enfermeiro deve possuir, além do conhecimento prático-cuidativo, um embasamento teórico-científico e fundamentado que guie a prática profissional para a aderência do tratamento farmacológico e não farmacológico.
A Teoria do Autocuidado, desenvolvida por Dorothea Orem, permite inferir a importância que a prática da adesão à terapia seja realizada pela livre decisão da pessoa com IC para a melhoria do seu estado de saúde ${ }^{8}$. A adesão terapêutica envolve a compreensão do nível de aceitação existente para que a pessoa realize os comportamentos baseados nas orientações e prescrições da equipe de saúde ${ }^{9}$.

Aliado a isso, o cuidado de enfermagem ao paciente com insuficiência cardíaca torna-se necessário tendo em vista a importância do foco educativo e terapêutico diretamente com enfrentamento da condição promovendo uma melhoria no autocuidado objetivando o alcance de uma melhor qualidade de vida. O enfermeiro como protagonista no processo de educação em saúde deve buscar alternativas para promover a compreensão da IC atendendo a complexidade do cuidado evitando complicações e reduzindo as reinternações hospitalares ${ }^{10}$.

Com isso, o objetivo do estudo foi identificar, na literatura científica, as principais orientações de enfermagem para o autocuidado de pessoas com insuficiência cardíaca.

\section{Metodologia}

Trata-se de uma revisão integrativa da literatura realizada seguindo as seis etapas recomendadas: identificação do tema e elaboração da questão de pesquisa, estabelecimento de critérios de inclusão e exclusão, identificação dos estudos nas bases de dados, categorização da amostra selecionada, análise e discussão dos resultados e apresentação da revisão ${ }^{11}$. A busca foi norteada pela questão norteadora: "Quais são as orientações de enfermagem para o autocuidado de pacientes com insuficiência cardíaca abordadas na literatura científica?".

As bases de dados nacionais e internacionais determinadas para a busca dos estudos foram: Literatura Latino-Americana e do Caribe em Ciências da Saúde (LILACS), Medical Literature Analysis and Retrieval System Online (MEDLINE) via PubMed, Base de Dados em Enfermagem (BDENF) e a biblioteca virtual Scientific Eletronic Library Online (SCIELO). 
Para a busca das publicações, foram empregados os descritores estabelecidos no Medical Subject Heading (MeSH) - heart failure, selfcare e nursing - para a busca no PubMed e os Descritores em Ciências da Saúde (DeCS) da Biblioteca Virtual em Saúde (BVS): insuficiência cardíaca, autocuidado e enfermagem, utilizando o operador booleano "AND" para a cruzamento dos descritores.

Os critérios de inclusão estabelecidos foram artigos disponíveis na íntegra, publicados eletronicamente no período de 2009 a 2018 - recorte temporal determinado por selecionar artigos que utilizaram orientações, guidelines e outros materiais como a III Diretriz Brasileira de Insuficiência Cardíaca publicada em 2009, redigidos nas línguas português, inglês e espanhol e que atendessem a pergunta norteadora do estudo. Foram excluídos da amostra estudos duplicados, revisões, teses, dissertações e/ou relatos de experiências.

Inicialmente foram identificados 249 estudos nas bases de dados elencadas, sendo 175 na MEDLINE; 29 na LILACS; 24 na BDENF e 21 na SciELO. Utilizando os critérios de elegibilidade, os estudos duplicados foram considerados apenas uma vez, sendo excluídas suas versões repetidas em mais de uma base de dados. Foram excluídos aqueles que não atendiam aos critérios de inclusão (disponíveis na íntegra, publicados nas línguas português, inglês e/ou espanhol dentro do recorte estipulado), permanecendo 165 publicações.

Após isso, o título e o resumo dos artigos foram lidos e analisados e, em seguida, aqueles que não responderam à pergunta norteadora da revisão e estudos utilizando metodologias estabelecidas como critério para retirada da amostra foram excluídos. As 32 publicações remanescentes foram estudadas na íntegra e avaliadas onde 21 artigos foram incluídos na amostra desta revisão.

Os artigos foram posteriormente categorizados em um banco de dados conforme os autores, períodos, ano de publicação, tipo de estudo e o nível de evidência (NE). Para a classificação quanto ao NE utilizou-se: 1) Revisões sistemáticas ou metanálise de ensaios clínicos randomizados relevantes; 2) Evidências provenientes de, pelo menos um, ensaio clínico randomizado controlado bem delimitado; 3) Ensaios clínicos bem delimitados sem randomização; 4) Estudos de corte e de caso-controle; 5) Revisões sistemáticas de estudos descritivos e qualitativos; 6) Evidências de um único estudo descritivo ou qualitativo; 7) Opiniões de autoridades ou relatório de comitês de especialistas ${ }^{12}$.

\section{Resultados}

Para a categorização da amostra, em relação ao ano de publicação, seis $(28,5 \%)$ artigos eram datados do ano de 2015, seguidos dos anos de 2017 (19\%), 2014 (14,2\%), 2016 (9,5\%) e 2012 (9,5\%).Quanto à origem, oito (38,1\%) são provenientes de periódicos internacionais e treze $(61,9 \%)$ de nacionais.

Detendo-se ao idioma, os estudos internacionais e os nacionais, com versão na língua inglesa disponível na íntegra, foram lidos no idioma inglês e os disponíveis apenas na versão nacional foram lidos em português. O nível de evidência de maior predominância encontrado nos estudos foi o nível $\mathrm{VI}$ presente em dezesseis $(76,2 \%)$ estudos e cinco $(23,8 \%)$ trabalhos apresentaram o nível de evidência II.

Com a revisão realizada, as principais orientações de enfermagem elencadas foram: Adesão farmacológica $(90,4 \%)$; Monitorização de sinais e sintomas (80,9\%); Restrição hidrossalina (66,6\%); Atividade física $(57,1 \%)$; Monitorização diária do peso $(52,3 \%)$; Hábitos alimentares (42,8\%); Conhecimento da insuficiência cardíaca (38,0\%); Vacinação (33,3\%); Suporte familiar (33,3\%); Consumo de álcool (28,5\%); Cessação do tabagismo (23,8\%); Repouso (14,2\%); Atividade sexual $(14,2 \%)$ e Atividade laboral $(4,7 \%)$.

Os artigos pertencentes à amostra foram caracterizados conforme os autores, ano, periódico, tipo de estudo empregado, nível de evidência e as orientações de enfermagem voltadas para os pacientes com insuficiência cardíaca estão apresentados no Quadro 1. 
Quadro 1. Caracterização dos artigos pertencentes a amostra da revisão quanto aos autores, ano, tipo de estudo, nível de evidência e orientações de enfermagem (2019)

\begin{tabular}{|c|c|c|}
\hline Autores / Ano & Tipo de Estudo (NE) & Orientações de Enfermagem \\
\hline $\begin{array}{l}\text { Bidwell, Vellrone, Lyons, } \\
\text { D'Agostino, Riegel, Juárez- } \\
\text { Vela, et al. / } 2015\end{array}$ & $\begin{array}{l}\text { Estudo descritivo e } \\
\text { transversal (VI) }\end{array}$ & $\begin{array}{l}\text { Adesão farmacológica e não-farmacológica; } \\
\text { Monitorização dos sinais e sintomas; Restrição de sódio e } \\
\text { Suporte familiar }\end{array}$ \\
\hline $\begin{array}{l}\text { Vourinen, Leppänen, } \\
\text { Kaijanranta, Kulju, Heliö, van } \\
\text { Gils,et al./2014 }\end{array}$ & $\begin{array}{l}\text { Ensaio Clínico } \\
\text { Randomizado (II) }\end{array}$ & Monitorização de sinais, sintomas e peso \\
\hline $\begin{array}{l}\text { Tawalbeh, Al Qadire, Ahmad, } \\
\text { Aloush, Abu Sumaga, Halabi } \\
\text { / } 2017\end{array}$ & $\begin{array}{l}\text { Estudo descritivo e } \\
\text { transversal (VI) }\end{array}$ & $\begin{array}{l}\text { Monitorização dos sinais, sintomas e peso; Adesão } \\
\text { farmacológica; Hábitos alimentares; Restrição } \\
\text { hidrossalina; Atividade física; Cessação do tabagismo }\end{array}$ \\
\hline $\begin{array}{c}\text { Cameron, Rhodes, Ski, } \\
\text { Thompson / } 2015\end{array}$ & Estudo qualitativo (VI) & $\begin{array}{l}\text { Adesão farmacológica; Restrição hidrossalina; Atividade } \\
\text { física; Suporte familiar }\end{array}$ \\
\hline $\begin{array}{l}\text { Köberich, Lohrmann, Mittag, } \\
\text { Dassen / } 2015\end{array}$ & $\begin{array}{l}\text { Ensaio Clínico } \\
\text { Randomizado (II) }\end{array}$ & $\begin{array}{l}\text { Conhecimento da IC; Restrição hidrossalina; Atividade } \\
\text { física; Cessação do tabagismo; Monitorização de sinais, } \\
\text { sintomas e peso; Hábitos alimentares; Atividade sexual; } \\
\text { Consumo de álcool; Adesão farmacológica }\end{array}$ \\
\hline $\begin{array}{l}\text { Ross, Ohlsson, Blomberg, } \\
\text { Gustafsson / } 2015\end{array}$ & $\begin{array}{l}\text { Método misto - } \\
\text { Estudo quase- } \\
\text { experimental e } \\
\text { qualitativo (VI) }\end{array}$ & $\begin{array}{l}\text { Conhecimento da IC; Adesão farmacológica; Atividade } \\
\text { física }\end{array}$ \\
\hline $\begin{array}{c}\text { Oliveira, Cordeiro, Rocha, } \\
\text { Guimarães, Albuquerque / } \\
2017\end{array}$ & $\begin{array}{l}\text { Ensaio Clínico } \\
\text { Randomizado (II) }\end{array}$ & $\begin{array}{l}\text { Conhecimento da IC; Monitorização de sinais e sintomas; } \\
\text { Adesão farmacológica e não-farmacológica }\end{array}$ \\
\hline $\begin{array}{l}\text { Conceição, Santos, Santos, } \\
\text { Cruz / } 2015\end{array}$ & $\begin{array}{l}\text { Estudo descritivo e } \\
\text { transversal (VI) }\end{array}$ & $\begin{array}{l}\text { Adesão farmacológica; Restrição sódica; Cessação do } \\
\text { tabagismo; Consumo de álcool; Monitorização de sinais, } \\
\text { sintomas e peso }\end{array}$ \\
\hline $\begin{array}{l}\text { Mantovani, Ruschel, Souza, } \\
\text { Mussi, Rabelo-Silva / } 2015\end{array}$ & $\begin{array}{c}\text { Estudo experimental } \\
\text { antes-depois (VI) }\end{array}$ & $\begin{array}{l}\text { Restrição hidrossalina; Atividade física; Monitorização de } \\
\text { sinais e sintomas; Adesão farmacológica; Vacinação }\end{array}$ \\
\hline $\begin{array}{c}\text { Nascimento, } \\
\text { Püschel / } 2013 \\
\end{array}$ & $\begin{array}{c}\text { Estudo transversal } \\
(\mathrm{VI})\end{array}$ & $\begin{array}{l}\text { Adesão farmacológica; Monitorização do peso; Restrição } \\
\text { hidrossalina; Hábitos alimentares; Consumo de álcool }\end{array}$ \\
\hline $\begin{array}{l}\text { Correia, Mesquita, Singh, } \\
\text { Puigbonet, Garcia Rosa / } \\
2016 \\
\end{array}$ & $\begin{array}{l}\text { Estudo descritivo e } \\
\text { exploratório (VI) }\end{array}$ & $\begin{array}{l}\text { Adesão farmacológica; Monitorização de peso; Suporte } \\
\text { familiar }\end{array}$ \\
\hline $\begin{array}{c}\text { Machado, Wansing, Klein, } \\
\text { Moraes, Rabelo-Silva / } 2016\end{array}$ & $\begin{array}{l}\text { Estudo transversal } \\
(\mathrm{VI})\end{array}$ & $\begin{array}{l}\text { Restrição hidrossalina; Atividade física; Monitorização de } \\
\text { sinais e sintomas }\end{array}$ \\
\hline $\begin{array}{l}\text { Feijó, Ávila, Souza, Jaarsma, } \\
\text { Rabelo / } 2012\end{array}$ & $\begin{array}{l}\text { Estudo metodológico } \\
\qquad(\mathrm{VI})\end{array}$ & $\begin{array}{c}\text { Adesão farmacológica; Atividade física; Hábitos } \\
\text { alimentares; Monitorização de sinais, sintomas e peso; } \\
\text { Restrição hidrossalina }\end{array}$ \\
\hline Souza, Queluci / 2014 & $\begin{array}{l}\text { Estudo descritivo e } \\
\text { qualitativo (VI) }\end{array}$ & $\begin{array}{c}\text { Adesão farmacológica; Monitorização de sinais, sintomas } \\
\text { e peso; Suporte familiar; Hábitos alimentares; Restrição } \\
\text { hidrossalina; Repouso; Vacinação }\end{array}$ \\
\hline $\begin{array}{l}\text { Bertuzzi, Souza, Moraes, } \\
\text { Mussi, Rabelo / } 2012\end{array}$ & $\begin{array}{l}\text { Estudo experimental } \\
\text { antes-depois (VI) }\end{array}$ & $\begin{array}{c}\text { Adesão farmacológica; Monitorização de sinais, sintomas } \\
\text { e peso; Vacinação; Atividade física; Conhecimento da IC; } \\
\text { Restrição hidrossalina; Cessação do tabagismo; Consumo } \\
\text { de álcool }\end{array}$ \\
\hline $\begin{array}{l}\text { Rabelo, Mantovani, Aliti, } \\
\text { Domingues / } 2011\end{array}$ & $\begin{array}{l}\text { Estudo de validação } \\
\qquad(\mathrm{VI})\end{array}$ & $\begin{array}{c}\text { Adesão farmacológica; Conhecimento da IC; } \\
\text { Monitorização de sinais, sintomas e peso; Restrição } \\
\text { hidrossalina; Atividade física; Vacinação }\end{array}$ \\
\hline $\begin{array}{l}\text { Arruda, Pereira, Figueiredo, } \\
\text { Scofano, Flores, Cavalcanti / }\end{array}$ & $\begin{array}{l}\text { Ensaio clínico } \\
\text { randomizado (II) }\end{array}$ & $\begin{array}{l}\text { Adesão farmacológica; Conhecimento da IC; } \\
\text { Monitorização de sinais e sintomas; Vacinação; Atividade }\end{array}$ \\
\hline 2017 & & $\begin{array}{c}\text { física; Atividade Sexual; Repouso; Cessação do } \\
\text { tabagismo; Consumo de álcool; Hábitos alimentares }\end{array}$ \\
\hline $\begin{array}{l}\text { Cavalcante, Lima, Custódio, } \\
\text { Oliveira, Meneses, Oliveira, } \\
\text { et al. } / 2018\end{array}$ & $\begin{array}{l}\text { Estudo transversal e } \\
\text { analítico (VI) }\end{array}$ & $\begin{array}{l}\text { Conhecimento da IC; Adesão farmacológica; Hábitos } \\
\text { alimentares; Suporte familiar; Monitorização sinais e } \\
\text { sintomas; Restrição hidrossalina; Atividade laboral; } \\
\text { Atividade Sexual; Cessação do tabagismo; Consumo de } \\
\text { álcool }\end{array}$ \\
\hline Linn, Azollin, Souza / 2016 & $\begin{array}{l}\text { Estudo longitudinal } \\
\text { retrospectivo }(\mathrm{VI})\end{array}$ & $\begin{array}{l}\text { Adesão farmacológica; Hábitos alimentares; Atividade } \\
\text { física; Monitorização de sinais, sintomas; Restrição hídrica }\end{array}$ \\
\hline $\begin{array}{l}\text { Liljeroos, Ågren, Jaarsma, } \\
\text { Stromberg / } 2017\end{array}$ & $\begin{array}{l}\text { Estudo descritivo e } \\
\text { qualitativo }(\mathrm{VI})\end{array}$ & $\begin{array}{c}\text { Conhecimento da IC; Atividade física; Adesão } \\
\text { farmacológica; Restrição hidrossalina; Monitorização de } \\
\text { sinais e sintomas; Vacinação; Suporte familiar }\end{array}$ \\
\hline $\begin{array}{l}\text { Clark, McDougall, Riegel, } \\
\text { Joiner-Rogers, Innerarity, } \\
\text { Meraviglia, et al. / } 2015\end{array}$ & $\begin{array}{l}\text { Ensaio clínico } \\
\text { randomizado (II) }\end{array}$ & $\begin{array}{c}\text { Adesão farmacológica; Monitorização de sinais, sintomas } \\
\text { e peso; Suporte familiar; Hábitos alimentares; Restrição } \\
\text { hidrossalina; Repouso; Vacinação }\end{array}$ \\
\hline
\end{tabular}

Legenda: NE - Nível de Evidência

Fonte: Elaborado pelo autor (2019). 


\section{Discussão}

A adesão à terapia farmacológica consistiu do tópico de larga abordagem dentre a amostra. Um estudo realizado com enfermeiros de uma enfermaria cardiológica de um hospital universitário traz nos discursos dos profissionais, que a terapêutica farmacológica constitui-se o fator mais preocupante no tratamento. Os relatos pautam-se na necessidade das práticas adequadas envolvendoo entedimento da importância da regularidade, horário e dose certa das medicações certas ${ }^{13}$.

Além disso, o uso de classes medicamentosas que possam contribuir para descompensações cardíaca, como medicamentos para Diabetes Mellitus e Hipertensão Arterial, deve ser categoricamente esclarecido pelo enfermeiro durante as orientações ofertadas ${ }^{14-15}$.

Os estudos salientam que a dificuldade e/ou inadequação da adesão farmacológica é influenciada por inúmeros fatores associados a prática terapêutica como baixa escolaridade, condições psicológicas e a complexidade do tratamento envolvendo a quantidade de medicações, seus custos e os efeitos ${ }^{7-16}$. Para o fortalecimento da adesão farmacológica o enfermeiro deve compreender os determinantes socioeconômicos e clínicos nos quais os pacientes com insuficiência cardíaca estão inseridos, entendendo que tal orientação constitui-se de um dos focos primordiais durante os cuidados de enfermagem ofertados.

Outra orientação envolve a monitorização dos sinais e sintomas característicos da IC, possibilitando ao enfermeiro acompanhar o processo evolutivo da condição em relação à melhora, piora ou estabilidade ${ }^{16}$. Uma intervenção com 153 pacientes, utilizando um questionário acerca do conhecimento da IC e do autocuidado, apresentou que $77 \%$ da amostra possuia o conhecimento da identificação dos sintomas, porém destes, grande parte, desconhecia como relacionar descompensações com sinais indicativos de piora clínica ${ }^{17}$.

A restrição hidrossalina é um aspecto de fundamental seguimento às recomendações profissionais, pois o controle do sódio configura-se como uma das orientações que trazem maior impacto familiar e/ou social. Entende-se que, pelas limitações causadas, os familiares e/ou cuidadores, em grande parte dos casos, são responsáveis pelo preparo das refeições ${ }^{18}$. Reitera-se que estratégias de auxílio ao profissional como táticas para o controle pela leitura e análise de rótulos de produtos solidificam a importância da adesão à mudança dos hábitos alimentares ${ }^{18-19}$.

Na mesma perspectiva, a restrição hídrica requer reforço educativo nos cuidados aos pacientes. Uma pesquisa realizada com 82 pacientes com IC em um hospital terciário referência em cardiologia utilizando a Escala de Autocuidado para Pacientes com Insuficiência Cardíaca (EAC-IC), obteve um índice de 48,8\% de afirmação, pelos entrevistados, ser improvável ou pouco provável a redução da ingesta líquida ${ }^{20}$.

No aspecto da alimentação, o controle dietético por meio de hábitos como estímulo a alimentação saudável é constantemente incitado para a melhoria da qualidade de vida. Orientações de enfermagem como evitar alimentos enlatados, industrializados e condimentados são algumas recomendações importantes que podem ser fornecidas aos pacientes ${ }^{21}$. Além do mais, permanancendo no aspecto nutricional, a manutenção e controle do peso corporal, por meio da monitorização diária, auxilia no manejo da insuficiência cardíaca. Um estudo realizado com 120 pacientes com IC destacou que, dentro das orientações de autocuidado, apenas $28 \%$ dos pacientes receberam orientações sobre o controle do peso destacando a fragilidade da abordagem desta pelos profissionais ${ }^{22}$.

Em síntese, os aspectos pertinentes à restrição hidrossalina e controle do peso são abordados na Diretriz Brasileira de Insuficiência Cardíaca Crônica e Aguda trazendo a recomendação da utilização diária de até 7 gramas de cloreto de sódio e o não estabelecimento de orientações específicas voltadas para a restrição hídrica e guias dietéticos ${ }^{2}$. O enfermeiro, embasado cientificamente na literatura analisada, detem-se na realização e reforço das orientações de forma a compreender a individualidade terapêutica.

No aspecto do cuidado orgânico, a atividade física é vista como um mecanismo de melhoria da tolerância à atividade apesar da baixa adesão entre os pacientes com IC. Tal fator relaciona-se com a possibilidade da exarcebação dos sintomas característicos da 
condição, além da necessidade de um acompanhamento profissional com a utilização de estratégias que estimulem a prática de atividade física ${ }^{23}$. Orientações de enfermagem pertinentes aos pacientes com IC envolvem caminhadas leves em locais planos, reforçando a necessidade do descanso e parada em caso de sinais de desconforto para evitar exarcebações ${ }^{24}$.

A literatura apresenta que um dos principais determinantes para a adesão terapêutica correta é a compreensão da condição, visto que desconhecimento é a principal barreira cognitiva para a adesão do autocuidado e das mudanças dos hábitos ${ }^{25}$. As pessoas com insuficiência cardíaca podem apresentar inúmeros questionamentos acerca da condição, podendo não compreender como a mesma se relaciona etiologicamente com o estresse e/ou hábitos de vida inadequados ${ }^{26}$. Torna-se necessária a utilização de mecanismos que propiciem uma compreensão efetiva e adequada de todos os aspectos pertinentes à condição pelo paciente e acompanhante ${ }^{27}$.

Uma recomendação constantemente presente, porém pouco aderida entre os pacientes com IC, é a vacinação anual contra a gripe. É nótorio que as pessoas demonstram estar cientes da relevância desta medida, mas o reforço continua sendo necessário ${ }^{28}$. Uma pesquisa realizada com 57 pacientes com IC internados buscou analisando influências sociais nos comportamentos de autocuidado apresentou que o esquema vacinal atualizado foi considerado satisfatório em $64,9 \%$ dos avaliados, sendo influenciado diretamente pelo suporte familiar ${ }^{29}$.

O suporte familiar e/ou social é uma das variáveis de real significância dentro do aspecto do autocuidado impactando na motivação dos pacientes em realizar as modificações do estilo de vida ${ }^{30}$. Pelo público-alvo prevalente da condição ser uma população na faixa etária acima de 65 anos e com um nível baixo de escolaridade, o auxílio familiar e/ou de cuidadores para a compreensão adequadas das orientações e monitorização clínica tornam-se aliadas em evitar descompensações e hospitalizações ${ }^{31-32}$.
Utilizando o envolvimento familiar e social dentro da perspectiva de adpatção às mudanças ocasionadas pela IC, o enfermeiro pode consolidar as orientações quanto a participação efetiva da rede de apoio ao paciente para que o impacto das transformações decorrentes da condição não afetem a adesão teraupêutica adequada.

A restrição do álcool e cessação do tabagismo correspondem à medidas não-farmacológicas intensivamente debatidos com as pessoas com insuficiência cardíaca. A orientação de enfermagem voltada a esses tópicos debruça-se na cessação total do fumo enquanto que o uso de álcool, se não for abstendido completamente, deverá ao menos ser reduzido para valores estabelecidos em $\leq 10 \mathrm{~mL}$ para mulheres $\mathrm{e} \leq$ $20 \mathrm{~mL}$ para homens $\mathrm{s}^{2-33}$.

Recomendações acerca da atividade sexual, atividade laboral e repouso, que foram menos abordadas na amostra, devem ser fortalecidas junto aos pacientes. Em relação à atividade laborativa, o seguimento da realização do trabalho deve ser individualizada mediante à estabilidade dos sintomas e da adesão correta da terapêutica ${ }^{2}$. Quanto ao repouso, deve-se reiterar o cuidado ao realizar um repouso prolongado pela possibilidade de diminuição da tolerância as atividades como, por exemplo, a ativdadade sexual $^{2}$. A prática sexual pode ser impossibilitada pelos sintomas da IC e/ou por alterações causadas por alguns medicamentos e, caso isso ocorra, a orientação adequada é buscar apoio na equipe de saúde para adequação da terapêutica² .

Denota-se com a revisão realizada que as orientações de enfermagem para a a pessoa com insuficiência cardíaca são aliados a adesão terapêutica voltadas ao autocuidado. Para a enfermagem,parafreseando a teorista Orem, influenciar a participação ativa do indíviduo dentro do seu processo de autocuidado, alicerçado teorica e cientificamente, visa trazer significativas modificações na qualidade de vida $^{8}$. A necessidade do reforço das recomendações pauta-se na concepção das demandas vistas para a promoção de um autocuidado de forma eficaz onde se torna possível a identificação dos pontos que carecem de um abordagem integral ${ }^{8}$. 
Portanto, percebe-se que o enfermeiro deve possuir um olhar diferenciado entendendo a heterogeneidade do público para adaptar as recomendações realizadas buscando romper com os obstáculos encontrados para a mudança do estilo de vida, favorecendo a busca de novas perspectivas para a melhoria do cuidado.

Destaca-se como limitação do estudo a escassez de literatura voltada diretamente ao autocuidado da pessoa com insuficiência cardíaca na perspectiva de orientações realizadas por enfermeiros. Notou-se que, comumente nos artigos obtidos inicialmente, as recomendações e intervenções destinavam-se a outros profissionais de saúde, evidenciando a necessidade da enfermagem apoderar-se da relevância do seus saberes e conhecimento técnico-científico frente à assistência desta população.

\section{Conclusão}

Conclui-se, com a presente revisão, que as principais orientações de enfermagem voltadas as pessoas com insuficiência cardíaca foram identificadas na literatura científica. Os estudos apontam uma correlação direta da prática adequada do autocuidado com uma redução significativa em taxas de reinternações e descompensações clínicas da condição.

Ressaltam que as informações repassadas pelo profissional promovem o estreitamento do vínculo com o paciente entendendo que os cuidados educativos de enfermagem interferem diretamente na adesão à terapêutica farmacológica e não-farmacológica em decorrência de um melhor acompanhamento da evolução da insuficiência cardíaca.

Porém, apesar das inúmeras recomendações, os índices de morbimortalidade da IC ainda se encontram elevados, evidenciando a necessidade de estratégias mais eficazes para melhorar a prática do autocuidado. A utilização de mecanismos educativos ancorados em metodologias ativas e/ou uso de tecnologias em saúde podem incorporar na consolidação das orientações fornecidas pelo enfermeiro durante à consulta.
Por fim, acredita-se que o enfermeiro deve-se apropriar cada vez mais do seu aspecto de educador, embasado cientificamente pelos estudos e diretrizes, para que a assistência prestada consiga preencher as lacunas existentes para a autonomia da pessoa com insuficiência cardíaca frente ao seu processo de autocuidado.

\section{Contribuições das autoras}

Silva AVC participou da concepção do estudo; decisão do percurso metodológico; busca, interpretação e análise dos estudos amostrais e redação do artigo científico. Carvalho BL participou da coleta de dados do estudo; categorização e interpretação dos dados obtidos e na redação do artigo científico. Guedes MVC participou na orientação do estudo; revisão crítica do objetivo do estudo; análise dos resultados; redação do artigo científico e aprovação da versão final.

\section{Conflitos de interesses}

Nenhum conflito financeiro, legal ou político envolvendo terceiros (governo, empresas e fundações privadas, etc.) foi declarado para nenhum aspecto do trabalho submetido (incluindo, mas não se limitando a subvenções e financiamentos, participação em conselho consultivo, desenho de estudo, preparação de manuscrito, análise estatística, etc.).

\section{Referências}

1. Souza MP, Araújo SM, Dourado MB, Gama GGG. Perfil epidemiológico de idosos com insuficiência cardíaca na unidade de terapia intensiva. Rev Enferm Contemporânea. 2017;6(1):42-8. doi: 10.17267/2317-3378rec.v6i1.1164

2. Rohde LEP, Montera MW, BocchiEA, Clausell NO, Albuquerque DC, Rassi S et al. Diretriz Brasileira de Insuficiência Cardíaca Crônica e Aguda. Arq Bras Cardiol. 2018;111(3):436-539. doi: 10.5935/abc. 20180190

3. Ministério da Saúde. Sistema de informações hospitalares do SUS, 2008-5. Sistema de informações sobre mortalidade 19962016. [Internet]. [acesso 2018 jul 05]. Disponível em: http://www. datasus.gov.br

4. Ministério da Saúde. Sistema de informações hospitalares do SUS, 2008-2018. [Internet]. [acesso 2018 jul 17]. Disponível em: http://www.datasus.gov.br 
5. Araújo AA, Nóbrega MML, Garcia TR. Diagnósticos e intervenções de enfermagem para pacientes portadores de insuficiência cardíaca congestiva utilizando a CIPE®. Rev Esc Enferm USP. 2013;47(2):385-92. doi: 10.1590/S008062342013000200016

6. Bocchi EA, Braga FGM, Ferreira SMA, Rohde LEP, Oliveira WA Almeida DR et al. III Diretriz Brasileira de Insuficiência Cardíaca Crônica. Arq Bras Cardiol. 2009;93(1 supl 1):1-71. doi: 10.1590/ S0066-782X2009002000001

7. Mantovani VM, Ruschel KB, Souza EN, Mussi C, Rabelo-Silva ER. Adesão ao tratamento de pacientes com insuficiência cardíaca em acompanhamento domiciliar por enfermeiros. Acta Paul Enferm. 2015;28(1):41-7. doi: 10.1590/1982-0194201500008

8. Orem DE. Nursing: concepts of practice. 6.ed. St. Louis: MosbyYear Book; 2001.

9. Liberato SMD, Souza AJG, Gomes ATL, Medeiros LP, Costa IKF, Torres GV. Relação entre adesão ao tratamento e qualidade de vida: revisão integrativa de literatura. Rev Elet Enf. 2014;16(1):1918. doi: 10.5216/ree.v16i1.22041

10. Gonçalves FG, Albuquerque DC. Educação em saúde de pacientes portadores de insuficiência cardíaca. Rev Enferm UERJ. 2014;22(3):422-8.

11. Cerqueira ACDR, Cardoso MVLML, Viana TRF, Lopes MMCO. Revisão integrativa de literatura: sono em lactentes que frequentam creche. Rev Bras Enferm. 2018; 71(2):424-30. doi: 10.1590/0034-7167-2016-0480

12. Galvão CM. Níveis de evidência. Acta Paul Enferm. 2006;19(2). doi: 10.1590/S0103-21002006000200001

13. Souza PMBB, Queluci GC. A arte de cuidar em pacientes com insuficiência cardíaca na alta hospitalar: considerações para a prática assistencial na enfermagem. Rev Pesq Cuid Fundam. 2014;6(1):153-67. doi: 10.9789/2175-5361.2014v6n1p153

14. Oliveira JEP, Montenegro Júnior RM, Vencio S, Foss-Freitas MC. Diretrizes da Sociedade Brasileira de Diabetes 2017-2018. São Paulo (SP): Editora Clannad; 2017.

15. Malachias MVB, Póvoa RMS, Nogueira AR, Souza D, Costa LS, Magalhães ME et al. $7^{a}$ Diretriz Brasileira de Hipertensão Arterial. Arq Bras. Cardiol. 2016;107(3):supl 3. doi: 10.5935/abc.20160153

16. Arruda CS, Pereira JMV, Figueiredo LS, Scofano BS, Flores PVP, Cavalcanti ACD. Efeito do grupo de orientação em pacientes com insuficiência cardíaca crônica: ensaio clínico randomizado. Rev Latino-Am Enferm. 2017;25:e2982. doi: 10.1590/15188345.2167 .2982
16. Vourinen AL, Leppänen J, Kaijanranta $H$, Kulju M, Heliö $\mathrm{T}$, van Gils $\mathrm{M}$ et al. Use of home telemonitoring to support multidisciplinary care of heart failure patients in Finland: randomized controlled trial. J Med Internet Res. 2014;16(12): e282. doi: 10.2196/jmir.3651

17. Rabelo ER, Mantovani VM, Aliti GB, Domingues FB. Adaptação transcultural e validação de um questionário de conhecimento de doenças e autocuidado, para uma amostra da população brasileira de pacientes com insuficiência cardíaca. Rev Latino-am Enferm. 2011;19(2):08 telas. doi: 10.1590/S010411692011000200008

18. Clark AP, McDougall G, Riegel B, Joiner-Rogers G, Innerarity S, Meraviglia $M$ et al. Health status and self-care outcomes following an education-support intervention for people with chronic heart failure. J cardiovasc nurs. 2015;30(4 supl 1):S3-13. doi: 10.1097/ JCN.0000000000000169

19. Cameron J, Rhodes KL, Ski CF, Thompson DR. Carers' views on patient self-care in chronic heart failure. J Clin Nurs. 2016;25(12):144-52. doi: $10.1111 /$ jocn.13124

20. Linn AC, Azollin K, Souza EN. Association between self-care and hospital readmissions of patients with heart failure. Rev Bras Enferm. 2016;69(3):500-6. doi: 10.1590/0034-7167.2016690312i

21. Machado CGD, Wansing GB, Klein C, Moraes MAP, Rabelo-Silva ER. Conhecimento do enfermeiro sobre insuficiência cardíaca em hospital geral. Rev Enferm UFSM. 2014;4(4):710-17. doi: $\underline{10.5902 / 2179769211633}$

22. Nascimento HR, Püschel VAA. Ações de autocuidado em portadores e insuficiência cardíaca. Acta Paul Enferm. 2013;26(6):601-7. doi: 10.1590/S0103-21002013000600015

23. Conceição AP, Santos MA, Santos B, Cruz DALM. Autocuidado de paciente com insuficiência cardíaca. Rev Latino-Am. Enferm. 2015;23(4):578-86. doi: 10.1590/0104-1169.0288.2591

24. Oliveira SKP, Lima FET. Validação de conteúdo da escala de avaliação do autocuidado de pacientes com insuficiência cardíaca. Rev RENE. 2017;18(2):148-55. doi: 10.15253/21756783.2017000200002

25. Liljeroos M, Ågren S, Jaarsma T, Stromberg A. Dialogues between nurses, patients with heart failure and their partners during a dyadic psychoeducational intervention: a qualitative study. BMJ Open. 2017;7:e018236. doi: 10.1136/ bmjopen-2017-018236

26. Ross A, Ohlsson U, Blomberg K, Gustafsson M. Evaluation of an intervention to individualize patient educational a nurse-led heart failure clinic: a mixed-method study. J Clin Nurs. 2015;24(1112):1594-602. doi: $10.1111 /$ jocn.12760 
27. Bertuzzi D, Souza EN, Moraes MA, Mussi C, Rabelo ER. Conhecimento do paciente com insuficiência cardíaca no contexto domiciliar: estudo experiemental. Online Braz J Nurs. 2012;11(3):572-82.

28. Feijó MK, Ávila CW, Souza EN, Jaarsma T, Rabelo ER. Adaptação transcultural e validação da European Heart Failure Self-care Behaviour Scale para o português do Brasil. Rev Latino-Am Enferm. 2012;20(5):988-96. doi: 10.1590/S010411692012000500022

29. Cavalcante LM, Lima FET, Custódio IL, Oliveira SKP, Meneses LST, Oliveira ASS et al. Influence of socio-demographic characteristics in the self-care of people with heart failure. Rev Bras Enferm. 2018;71(supl 6):2604-1. doi: 10.1590/0034-71672017-0480

30. Tawalbeh LI, Al Qadire M, Ahmad MM, Aloush S, Abu Sumaga Y, Halabi M. Knowledge and self-care behaviors among patients with heart failure in Jordan. Res Nurs Health. 2017;40(4):350-9. doi: 10.1002/nur.21805

31. Bidwell JT, Vellone E, Lyons KS, D'Agostino F, Riegel B, JuárezVela $R$ et al. Determinants of heart failure self-care maintenance and management in patients and caregivers: A dyadic analysis. Res Nurs Health. 2015;38(5):392-402. doi: 10.1002/nur.21675

32. Correia DMS, Mesquita ET, Singh M, Puigbonet ME, Garcia Rosa MLG. Challenges for the heart failure care: exploratory research with nurses in Ontario. Journal of Research: Fundamental Care Online. 2016;8(4):5150-5. doi: 10.9789/21755361.2016.v8i4.5150-5155

33. Köberich S, Lohrmann C, Mittag O, Dassen T. Effects of a hospital-based education programme of self-care behaviour, care dependency and quality of life in patients with heart failure - a randomized controlled trial. J Clin Nurs. 2015;24(11-12):1643-55. doi: $10.1111 /$ jocn.12766 